\title{
Mental Health Literacy in Zurich: A First Measurement Attempt Using the General HLS-EU-Q47
}

\author{
Michael Schneider ${ }^{1,2}$, Rebecca Jaks ${ }^{2}$, Daniela Nowak-Flück ${ }^{2}$, Dunja Nicca $^{3}$ and \\ Saskia Maria De Gani ${ }^{2 *}$ \\ 1 Department of Health Sciences and Technology, Institute of Human Movement Sciences and Sport, Eidgenössische \\ Technische Hochschule Zurich, Zurich, Switzerland, ${ }^{2}$ Health Literacy Division, Careum Foundation, Zurich, Switzerland, \\ ${ }^{3}$ Epidemiology, Biostatistics and Prevention Institute, University of Zurich, Zurich, Switzerland
}

Background: Mental health literacy (MHL) promises to be an important factor for public health by enabling people to take responsibility for their own mental health. To date, there is no measurement tool that allows the assessment of a comprehensive understanding of $\mathrm{MHL}$ as part of health literacy $(\mathrm{HL})$. Nonetheless, the widely used Health Literacy Survey European Questionnaire 47 (HLS-EU-Q47) includes items assessing at least some MHL-aspects in the context of $\mathrm{HL}$. The present study aimed at investigating how these $\mathrm{MHL}$-aspects are related to $\mathrm{HL}$, health behavior and health outcome and how they differ between sociodemographic groups.

Methods: Data from the Health Literacy Survey Zurich 2018, collected by an adapted version of the HLS-EU-Q47, served to investigate these relationships.

Results: $\mathrm{MHL}$-aspects were related to $\mathrm{HL}$, health behavior and health outcome. Nearly half of all respondents (45\%; $N=904)$ showed low $M H L$ levels, particularly those with higher age and higher financial deprivation.

Conclusions: Relations of MHL-aspects with $\mathrm{HL}$, health behavior, and health outcome indicate their potential importance for future interventions in public health, addressing mental health and MHL. A specific MHL tool is needed to comprehensively investigate these relations, which could be developed by extending the present measurement approach.

Keywords: mental health literacy, health literacy, measurement tool, health behavior, health outcome

\section{INTRODUCTION}

Mental health is an essential requirement for good health. Therefore, it is an integral vision of the World Health Organization (WHO) to achieve the highest possible standard of mental health and well-being for the entire population (1). Nowadays, mental health conditions cause one fifth of all years lived with disability worldwide (1), and have a significant impact on the quality of life of the affected individuals and their families (2). In Switzerland, 15\% of the population report moderate to severe mental stress, while around three quarters of those with severe mental stress or depressive symptoms suffer from physical complaints as well (3). Considering that respondents of the Swiss Corona Stress Study (4) reported an increase of stress and depressive symptoms during the COVID-19 pandemic, the psychological burden of the Swiss population may even be higher. 
Mental health literacy (MHL) promises to be an important resource to cope with this burden, as it may not only facilitate recognition of mental disorders and early help-seeking (5), but possibly also promote mental health (6).

MHL can be considered as an integral part of health literacy (HL), which itself can be understood as an individual's motivation, knowledge and ability to find, understand, and use health information to manage one's own health through informed decisions and corresponding health behavior (7). Hence, HL focuses on competencies in dealing with health information and exceeds aspects only linked to disease management. In fact, HL also includes the two dimensions disease prevention and health promotion that are important for both mental and general health. As the concept of HL is still discussed diversely $(7,8)$, also MHL has been explored with different definitions so far $(9,10)$. A common definition states that MHL includes the ability to recognize specific mental disorders, knowledge of risk factors, causes, self-treatments, availability of professional help, knowledge on how to seek mental health information as well as attitudes promoting recognition and appropriate help-seeking (11). Additionally, there have been discussions on an extended definition of MHL that does not only include knowledge and beliefs about mental disorders (12-14). Accordingly, Kutcher et al. (13) defined four main components of MHL: (1) understanding how to obtain and maintain positive mental health, (2) understanding mental disorders and their treatments, (3) decreasing stigma related to mental disorders, and (4) enhancing help-seeking efficacy, which means knowing when and where to seek help and developing competencies designed to improve one's mental healthcare and self-management capabilities. Including the understanding of how to obtain and maintain a good mental health in the definition of MHL is in line with the comprehensive concept of HL as well as the WHO's definition of mental health (15), i.e., mental health is more than the absence of mental health disorders.

The identification of specific sociodemographic groups reporting low MHL levels is important for the initiation of targeted interventions to strengthen their abilities to care for their own mental health. To identify whether people with a low level of MHL might also report needs concerning general health, it is also important to investigate their health behavior and health outcome. While many studies examined the relationship of HL and sociodemographic factors $(16,17)$, health behavior (16-19), and health outcome $(16,17,20)$, so far only few studies examined these aspects in relation to MHL. These studies related MHLaspects to sociodemographic characteristics such as age (21-23), gender (21-24), education (21-23), financial situation $(23,25)$, and rural residence $(24,26)$. Studies investigating MHL-aspects in the context of health behavior showed that stigma could be associated with more frequent alcohol and drug abuse (27), and low rates of help-seeking could be associated with higher rates of substance use disorders (28). In contrast, the few studies on MHL and health outcome showed that on the one hand higher

Abbreviations: HLS-CH-15, Swiss Health Literacy Survey; HLS-ZH-18, Health Literacy Survey Zurich.
MHL levels were related to better (self-assessed) health $(23,29)$, and on the other hand, inadequate MHL levels were associated with increased odds for moderate to severe depression (30). In summary, so far studies on MHL used only few measures of health behavior and health outcome and merely focused on specific subpopulations. Additionally, these studies were based on different definitions of MHL and mostly omitted the aspect of positive mental health.

In addition, these studies investigated MHL with different measurement tools (10). However, to date, no specific instrument can be found which assesses the comprehensive spectrum of MHL as part of general HL. Moreover, most studies so far have related MHL only to specific sociodemographic characteristics or few aspects of health behavior and health outcome. Nonetheless, the widely used instrument to assess general HL-the so-called Health Literacy Survey European Questionnaire 47 (HLS-EUQ47) (31)_includes at least some MHL-aspects in the context of HL. The HLS-EU-Q47 is usually applied to assess general HL including its specific abilities to access, understand, appraise and apply health information across the areas of healthcare, disease prevention and health promotion (16). Containing only few items that consider aspects of MHL, the questionnaire originally was not constructed to holistically assess MHL. However, it offers the opportunity to assess some MHL-aspects and their relation to $\mathrm{HL}$, several sociodemographic characteristics and aspects of health behavior and health outcome. Therefore, the aim of the present study was to make a first attempt to examine MHL in the population of Zurich using this instrument and the MHL-aspects as well as their relation to general HL, health behavior, health outcome, and sociodemographic characteristics. For this purpose, recent data from a study on general HL of the population of the canton of Zurich- "Health Literacy Survey Zurich" (HLS-ZH-18) (32)—was used.

\section{MATERIALS AND METHODS Study Population}

For the analysis, data of the population survey HLS-ZH-18 was used. Parts of the data have been analyzed and published in another context (32), other data has remained unpublished so far. The study population consisted of a total of 1,000 residents of the canton of Zurich (Switzerland) aged 18 years or older. Participants were interviewed between November and December 2018 using Computer Assisted Personal Interviews (CAPI) in German language. Data was collected by a third party (gfs.bern AG, research institute, Bern, Switzerland), which had also collected the data for the "Swiss Health Literacy Survey" (HLS-CH-15) (17). The present sample size was considered to be enough in order to conduct population- and subgroup analyses. Sampling error was 3.2. Sampling was conducted by a random selection of 100 cantonal sampling points and predefined quotas on site (age, gender). Communities with at least 1,000 residents built the basis for the sampling points. Larger communities had several sampling points (one for every 1,000 residents). The type of settlement was also taken into account when drawing the sampling points. A total of ten interviews per sampling point were conducted. Trained interviewers randomly interviewed 
TABLE 1 | MHL-associated items from the HLS-ZH-2018.

\begin{tabular}{ll}
\hline Item & $\begin{array}{l}\text { On a scale from very easy to very difficult, how easy would } \\
\text { you say it is to: }\end{array}$ \\
\hline Q4* "...find out where to get professional help when you are ill? \\
(doctor, pharmacist, and psychologist)" \\
Q18 "...find information on how to manage mental health problems like \\
stress or depression?" \\
Q33 "...find out about activities that are good for your mental \\
well-being? (meditation, exercise, walking, Pilates etc.)" \\
"“... how easy would you say it is to understand information on \\
how to keep your mind healthy?"
\end{tabular}

*Although Q4 can be related also to MHL, participants' answers to it may refer not only to help from a psychologist but also from a doctor or a pharmacist. This has to be considered when interpreting the data.

pedestrians, whereby interviewers were free to choose where they contacted the participants. The mean duration of the interview was $30.3( \pm 6) \mathrm{min}$. Participants were verbally informed about the goals, framework conditions and data protection measures before they gave their informed consent to participate in this study. All processes were in line with the legal and association requirements for the protection of data and personal rights (VSMS). A separate ethical approval for this study was not necessary.

\section{Questionnaire}

The HLS-ZH-18 questionnaire was based on the national survey HLS-CH-15 (17), which in turn consisted of the 47 adapted HL items of the HLS-EU-Q47 (31). All of these self-assessmentinstruments served to assess HL as well as health behavior, health outcome and sociodemographic characteristics.

\section{MHL-Associated Items}

The HLS-EU-Q47 and the HLS-CH-15 questionnaire do not contain a specific MHL module so far. Therefore, in the present study (HLS-ZH-2018), four items related to mental health or MHL, respectively, could be identified and are referred to as "MHL-associated items." These four items (Q4, Q18, Q33, and Q40) built the focus of the present study (Table 1).

\section{MHL-Index, General HL-Index, and HL-Index}

Out of the four MHL-associated items Q4, Q18, Q33, and Q40 an MHL-Index was built. As a second index, the general HL-Index $\left(\mathrm{HL}_{47}\right)$ including all $47 \mathrm{HL}$ items was built. The third index that was built was the HL-Index $\left(\mathrm{HL}_{43}\right)$ and included $43 \mathrm{HL}$ items, without the four MHL-associated items. All items were assessed with a Likert scale and numerical values were accordingly assigned ("very easy" = 4, "fairly easy" = 3, "fairly difficult" $=2$, "very difficult" =1). Based on these values, corresponding indices for each individual were built by calculating the mean and then applying the following formula, as recommended by the HLS-EU consortium $(16,33)$ :

$$
\text { Index }=(\text { mean }-1) \times \frac{50}{3}
$$

Accordingly, the indices were only calculated if a minimum respondent rate of $80 \%$ in all $47 \mathrm{HL}$ items was achieved and all four MHL-associated items were rated as well. These criteria resulted in the inclusion of 904 participants. In a novel approach, the here calculated MHL-Index was interpreted like the standard general HL-Index $\left(\mathrm{HL}_{47}\right)$, which means that $0-25$ points were rated as "inadequate," $>25-33$ as "problematic," $>33-42$ points as "sufficient" and $>42-50$ points as "excellent" MHL or HL, respectively $(16,33)$.

For the multiple logistic regression analysis, the MHLIndex was also defined dichotomously, whereby the categories "excellent" and "sufficient" ( $>33-50$ points) were summarized as "high MHL" and "problematic" and "inadequate" (0-33 points) were summarized as "low MHL".

Cronbach's alpha for all 47 items was 0.889 , indicating a high level of internal consistency. For MHL-associated items Cronbach's alpha was 0.547 .

\section{Sociodemographic Characteristics, Health Behavior, and Health Outcome}

Sociodemographic characteristics as well as health behavior and health outcome were assessed with the same questions (except for minor changes) and scales as in the HLS-CH-15 (17). Included sociodemographic variables were age, gender, education, financial deprivation, and type of settlement. Included health behavior variables were smoking behavior, alcohol consumption and physical exercise frequency. Body-mass-index (BMI), self-assessed health status and presence of chronic disease were included as health outcome variables.

For the multiple logistic regression analysis, several variables had to be re-categorized: (1) educational levels that have been classified according to the International Standard Classification of Education (ISCED) (34) were divided into the three categories low (level 0-2), medium (level 3-4) and high (level 5-6) education; (2) alcohol consumption was categorized as excessive (very excessive, excessive) or non-excessive (moderate, low, no alcohol); (3) physical exercise frequency was categorized into weekly (each day to a few times a week) and less than weekly (a few times per month to not at all); and (4) self-assessed health status was categorized as bad (very bad, bad), medium or good (good, very good).

\section{Data Analysis and Statistics}

Collected data were weighted according to the sociodemographic characteristics age/sex interlocked, type of settlement and highest level of education to account for the sample design, to adjust for respective sociodemographic characteristics and to increase representativity of the results. The Federal Statistical Office's statistics served as a reference for the weights $(35,36)$. Descriptive statistical analysis was used to characterize the sample, to analyze answer frequencies regarding MHL-associated items and to investigate MHL levels of the study population and their associations with $\mathrm{HL}_{47}$. Subgroup analysis with $<50$ respondents was-whenever possible-avoided. The interpretation of this analysis was almost impossible because of sampling errors of \pm 14 percentage points. Hence, when smaller subgroups were identified, this was explicitly pointed out. 
To investigate associations of $\mathrm{MHL}$ with $\mathrm{HL}_{47}, \mathrm{HL}_{43}$, and single HL items, spearman's rank correlation coefficients were calculated. In this context, $\mathrm{HL}_{43}$ was used to investigate the association of MHL and HL-thus HL independent of the four MHL-associated items. To evaluate the significance and directions of the associations between MHL and health behavior, health outcome and sociodemographic characteristics, spearman's rank correlation coefficients were calculated. For all spearman's rank correlation coefficients, respective variable scales were defined in ascending order. In a second step, associations of MHL (dependent variable) with health behavior, health outcome and sociodemographic characteristics (independent variables) were assessed in a multiple logistic regression model. This step allowed the comparison of the odds ratio (OR) of different subgroups for having low MHL levels. Corresponding listwise exclusion led to a sample of 831 respondents in total. Assumptions for all conducted statistical tests were fulfilled. The response category "do not know" was interpreted as a missing value. Respective tests were two-sided and for multiple logistic regression 95\% confidence intervals (CI) for OR were calculated.

Statistical analysis was conducted with the IBM SPSS v.26 software (IBM Corp. Armonk, NY, USA). For all statistical analyses, a value of $p<0.05$ was considered significant. Results are presented as mean \pm standard deviation (SD), percentage $(\%)$, spearman's rank correlation coefficient $\left(r_{s}\right), p$-value, OR, and CI.

\section{RESULTS}

\section{Characteristics of the Study Group}

Overall, 904 participants were included in the analysis. Study participants were $46.2 \pm 18.0$ years old. The youngest respondent was 18 and the oldest 88 years old. Sociodemographic characteristics of the included study population are presented in Table 2.

\section{MHL of the Population of Zurich}

The average MHL of men and women in Zurich was inadequate $(32.6 \pm 8.3)$. Accordingly, nearly half of all respondents showed a problematic or inadequate MHL (Figure 1).

Most difficulties (37\%) were reported with "...find information on how to manage mental health problems like stress or depression?” (Q18, Figure 2). Least difficulties (15\%) were reported with "...find out where to get professional help when you are ill? (doctor, pharmacist, and psychologist)" (Q4, Figure 2).

\section{MHL and HL}

Ninety-one percent of the participants with inadequate MHL showed inadequate or problematic $\mathrm{HL}_{47}$. In contrast, $88 \%$ of the participants with excellent MHL showed sufficient or excellent $\mathrm{HL}_{47}$ (Figure 3).

MHL significantly correlated to $\mathrm{HL}_{43}\left(r_{s}=0.563, p<0.001\right)$. In addition, MHL showed the strongest correlations with the $\mathrm{HL}_{43}$ items Q2, Q17, Q20, and Q32 (Table 3).
TABLE 2 | Sociodemographic characteristics of the included study population.

Total $(N=904)$

$\%(n)$

\begin{tabular}{lc}
\hline Gender & \\
Female & $51 \%(456)$ \\
Male & $49 \%(448)$ \\
Age & \\
$18-39$ & $37 \%(341)$ \\
$40-64$ & $42 \%(386)$ \\
$65+$ & $21 \%(177)$ \\
Education & \\
Low & $20 \%(66)$ \\
Medium & $43 \%(662)$ \\
High & $37 \%(176)$ \\
Type of settlement & \\
Rural & $5 \%(47)$ \\
Small/mid-sized city & $16 \%(156)$ \\
Big city & $80 \%(701)$
\end{tabular}

(n), unweighted number of cases; \%, weighted percentage of a total of 904 respondents. Percentages are rounded mathematically and do not always add up to exactly $100 \%$.

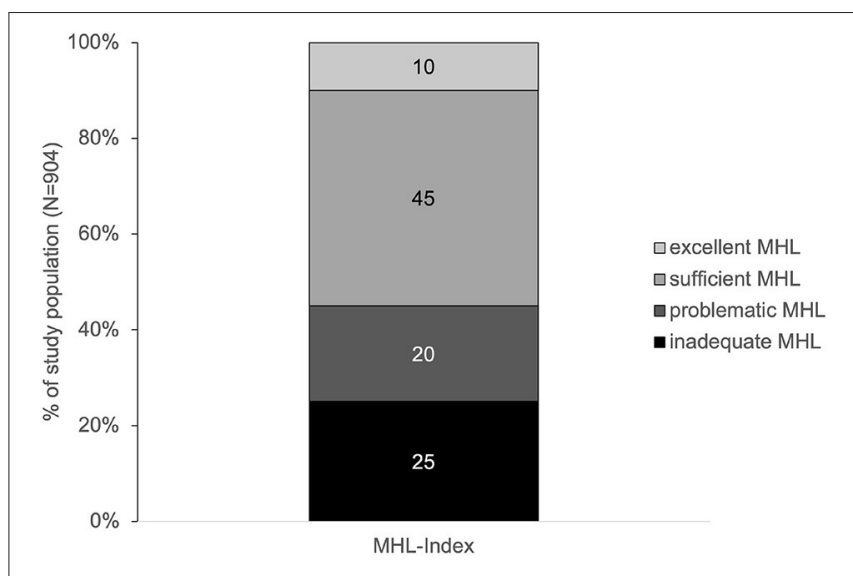

FIGURE 1 | MHL levels in percentage of the study population.

\section{MHL and Its Relations to Sociodemographic Characteristics, Health Behavior, and Health Outcome}

Compared to general HL, MHL showed correlations with the same direction but lower strength with all sociodemographic characteristics, except for rural residence. Hence, MHL was positively correlated to education $\left(r_{s}=0.167, p<0.001\right)$, and negatively correlated to age $\left(r_{s}=-0.173, p<0.001\right)$ as well as financial deprivation $\left(r_{s}=-0.307, p<0.001\right)$. No association was found between MHL and type of settlement $\left(r_{s}=0.012, p\right.$ $=0.688)$. Significant effect sizes were found for age and financial deprivation (Table 4): Participants aged 65 years and older (OR $=2.542$, 95\% CI: 1.509-4.282) and those with high financial deprivation ( $\mathrm{OR}=2.314,95 \% \mathrm{CI}: 1.560-3.432)$ were more than 


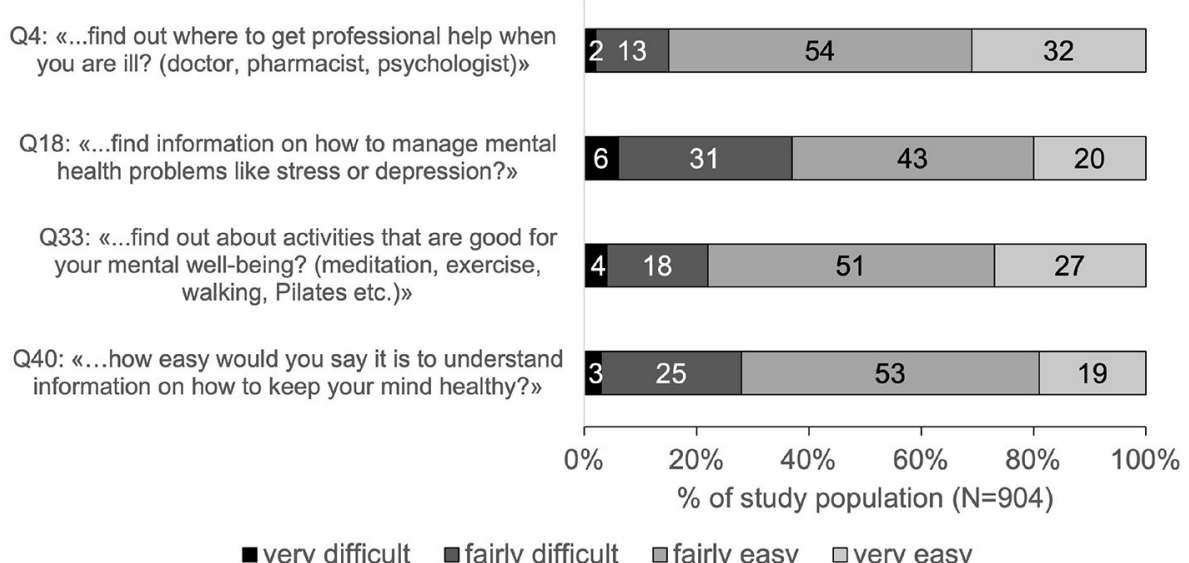

FIGURE 2 | Ratings of MHL-associated items in percentage of the study population. Percentages are rounded mathematically and do not always add up to exactly $100 \%$.

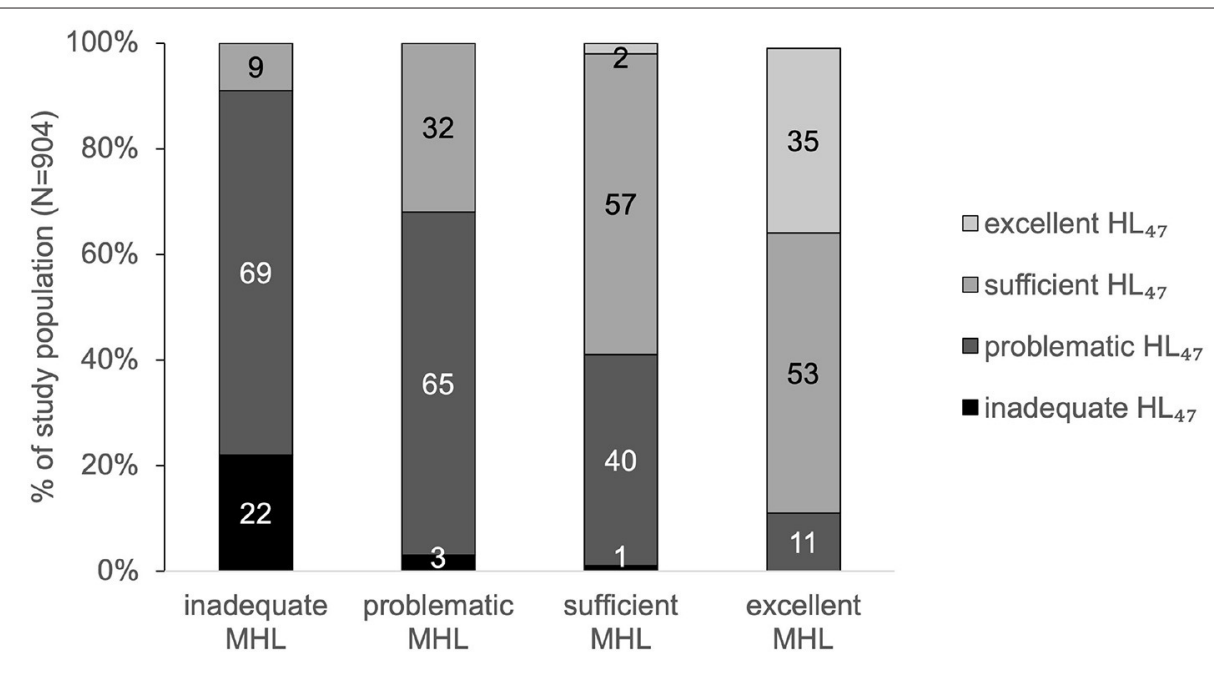

FIGURE 3 | Association of $\mathrm{MHL}$ and $\mathrm{HL}_{47}$.

twice as likely to have low MHL than younger residents and participants with low financial deprivation.

In addition, and again compared to general $\mathrm{HL}$, in the most cases MHL showed correlations of the same direction with health behavior and health outcome. Hence, MHL was positively correlated to physical exercise frequency $\left(r_{s}=0.254, p<0.001\right)$ and self-assessed health status $\left(r_{s}=0.263, p<0.001\right)$. In contrast, smoking behavior $\left(r_{s}=-0.130, p<0.001\right)$, BMI $\left(r_{s}=-0.066\right.$, $p<0.05)$, and occurrence of chronic disease $\left(r_{s}=-0.161, p<\right.$ $0.001)$ were negatively correlated with MHL. No association was found between MHL and alcohol consumption $\left(r_{s}=0.003, p=\right.$ $0.910)$. Significant effect sizes were found for physical exercise frequency, smoking behavior, and BMI (Table 4). Individuals who reported to be physically active less than once per week were more likely to have low MHL than their counterparts (OR = 2.214, 95\% CI: 1.532-3.200). Smokers and overweight individuals were more likely to have low MHL compared to non-smokers and individuals with normal weight.

\section{DISCUSSION}

Nearly half of the study population reported low MHL: $20 \%$ showed problematic and 25\% inadequate MHL. Thus, a substantial part of Zurich's population seems to have considerable difficulties with handling information on mental health. The main difficulty hereby concerned the access to information on how to cope with mental health problems. A similar result could be found in the Swiss national study HLS-CH-15 (17). Concerning MHL, also the Swiss population reported most difficulties in finding information on how to manage mental health problems like stress or depression (27\% in HLS-CH-15 vs. 
TABLE 3 | Analysis of the strongest correlations between MHL and single items of the $\mathrm{HL}_{47}$.

\begin{tabular}{|c|c|c|c|}
\hline Item & Item description & $r_{s}^{*}$ & $p$-value \\
\hline Q2 & $\begin{array}{l}\text { "... find information on treatments of } \\
\text { illnesses that concern you?" }\end{array}$ & 0.387 & $<0.001$ \\
\hline Q17 & $\begin{array}{l}\text { "... find information about how to } \\
\text { manage unhealthy behavior such as } \\
\text { smoking, low physical activity, and } \\
\text { drinking too much?" }\end{array}$ & 0.377 & $<0.001$ \\
\hline Q20 & $\begin{array}{l}\text { "... find information on how to prevent } \\
\text { or manage conditions like being } \\
\text { overweight, high blood pressure, or } \\
\text { high cholesterol?" }\end{array}$ & 0.400 & $<0.001$ \\
\hline Q32 & $\begin{array}{l}\text { "... find information on healthy } \\
\text { activities such as exercise, healthy } \\
\text { food and nutrition?" }\end{array}$ & 0.404 & $<0.001$ \\
\hline
\end{tabular}

*Spearman's rank correlation coefficient $\left(r_{s}\right)$

$37 \%$ in HLS-ZH-18). Considering these results, it seems crucial for the entire population to facilitate access to information on mental health. Knowing where to find information on coping strategies is a first step toward learning and applying such strategies to deal with mental health issues. This is even more important in respect of the increasing number of people with mental health problems (4) and other factors that could hinder help-seeking, like stigma for example (37) that seems to be still high in Switzerland (38). Furthermore, a lack of knowledge on strategies to deal with mental health problems has recently been detected in the younger Swiss population (39). This lack of knowledge could possibly also count for the general population. Thus, strengthening the access to information related to this knowledge seems to be of great necessity.

The present study population also showed difficulties in accessing and understanding information on the promotion and maintenance of their mental health. More than a fifth (22\%) reported difficulties with finding information about activities that are good for their mental wellbeing (Q33) and more than a quarter (28\%) reported difficulties with understanding information on how to keep their own mind healthy (Q40). Considering that during a pandemic like the COVID-19 pandemic, possibilities of mental health promoting activities, as for example meeting friends, or participating at community sports activities, might be restricted, it can be expected that finding appropriate mental health promoting activities might even be more difficult. In this context another recent Swiss survey concluded that there may not only be a lack of factual knowledge, but also concrete knowledge for action for mental health promotion (38). For example, only $46 \%$ of the respondents reported that they knew how to strengthen their mental health. Therefore, it seems to be important to not only offer alternative mental health promoting activities but also to make people aware of them, facilitate access to them, and increase the understanding of their importance. The current study as well as the Swiss national study on general HL showed that people report more difficulties with appraising and applying health information rather than with finding and understanding them.
Connecting this to the present findings, one might expect that if the assessment of MHL would have also included the two domains of appraising and applying information on mental health, MHL levels of the population might have even been lower and more problematic.

The results of this study indicate that MHL can be associated with general HL. Most of the respondents with inadequate MHL also showed inadequate or problematic general HL. This correlation was found to be true, irrespective of whether the four MHL-associated items were included into the model of $\mathrm{HL}\left(\mathrm{HL}_{47}\right)$ or not $\left(\mathrm{HL}_{43}\right)$. This indicates that people with low MHL often not only seem to have difficulties with finding and understanding information on mental health, but also with handling health information in general. People with low MHL therefore possibly may need to be supported not only in their abilities to care for their mental health but in a more comprehensive manner, including their physical health. Furthermore, the relation between MHL and HL seems to support the understanding of MHL as an integral part of HL. This relationship between MHL and HL needs to be carefully treated, however, as the questionnaire did mainly focus on general $\mathrm{HL}$ and did not include a comprehensive conceptualization of MHL, but a rather limited number of MHL-associated items. Nonetheless, the present findings are in accordance with another study that also showed a substantial association between MHL and HL (22). In addition to the present approach, the referred study considered HL as a predictor of MHL. The authors pointed out that poor HL could be associated with greater prevalence of mental illness symptoms and a lower likelihood to seek professional help for these symptoms. In the present study, however, HL is not understood as an antecedent for MHL or vice versa, as for example the ability to handle information on general health does not necessarily influence the ability to handle information on mental health. It is rather hypothesized that personal, situational, societal, and environmental determinants that have an influence on HL (7), may also determine MHL.

The present study found older age, lower education, and higher financial deprivation to be associated with low MHL and low HL. Low MHL in older and lower educated in Switzerland were also found in another survey which stated that they report more pronounced difficulties in understanding information on mental disorders (38). Reasons for low HL in these subgroups may at least also partly be responsible for low MHL. In other words, the pronounced difficulties with higher age regarding dealing with general health information as well as accessing and understanding information on mental health may be explained by an age-dependent decline of cognitive abilities (40). Furthermore, health information is increasingly often available online. Accessing this information and assessing the quality of online health information seems to be a great challenge, especially for the elderly (41). Another factor that might affect the access and understanding of information on mental health in general, but especially at higher age, is stigma. Actually, stigma has been seen as a significant barrier to access care in case of mental disorders in elderly people (42), whereby especially Swiss people over 80 years seem to be affected by stigmatization (38). In this context, the WHO, the World Psychiatric Association and the 
TABLE 4 | Associations of MHL and sociodemographic characteristics, health behavior, and health outcome.

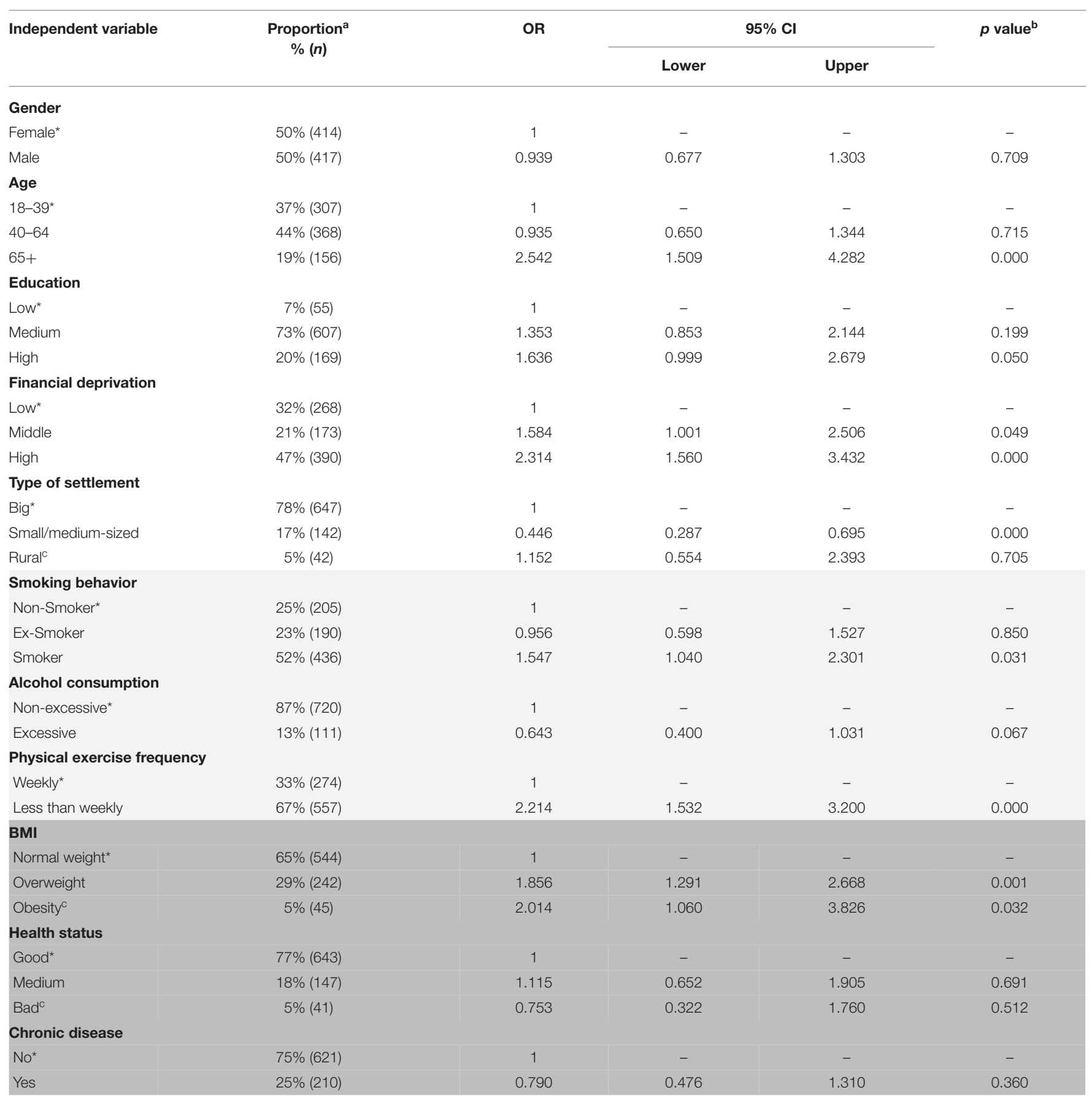

${ }^{a}(n)$, number of cases per subgroup; \%, percentage of a total of 831 included respondents.

${ }^{b} \mathrm{p}$-value for multiple logistic regression analysis with $\mathrm{MHL}$ as dependent variable [low vs. high $\mathrm{MHL}$ (=reference category)]

c Subgroup analysis with less than 50 respondents.

${ }^{*}$ Reference categories for odds ratio analysis.

Sociodemographic characteristics are colored white, health behavior with light gray and health outcome with dark gray.

Percentages are rounded mathematically and do not always add up to exactly $100 \%$.

Swiss Society for Public Health have emphasized the importance of destigmatization $(42,43)$. Assuming that stigmatization may have decreased (44), destigmatization is still ongoing, and awareness of mental health issues is rising, MHL could possibly profit thereof in the future. Apart from the present results, weak depressive symptoms (45) as well as medium to high mental stress seem to increase with the years after retirement (46). This further indicates the great need to strengthen MHL levels of the 
elderly. MHL of this population group could be strengthened by further decreasing stigma, increasing awareness of mental health issues as well as by facilitating and empowering them to access trustful and easy comprehensible (online) information on mental health.

Lack of awareness of mental health issues combined with stigma is also indicated in lower educated people (47-51), and may provide a possible explanation for their difficulties in accessing and understanding information on mental health. Respective subgroups showed higher levels of stigma (47), less knowledge (48), and poorer recognition of mental disorders (4951). The need to improve MHL of low educated people seems to be especially important as they are more affected by mental stress $(3,52)$ and common mental disorders $(53)$ than higher educated people. Besides low education, also high financial deprivation was associated with low MHL. This finding could be due to different reasons: Financially disadvantaged people may possibly less often use mental health services due to restricted access and financial reasons, and hence also be less aware of mental health issues. In addition, low financial capacities might also prevent them from participating in activities that could promote mental health.

Moreover, low HL was often found to be associated with poorer health behavior and outcome $(16,17,20)$, whereas high HL was associated with more favorable health behavior and outcome (16-19). Therefore, it was hypothesized that low MHL (as part of HL) would be similarly associated with less favorable health behavior and worse health outcome. Accordingly, positive health behavior (reflected by higher physical exercise frequency and less smoking) and better health outcome (reflected by higher self-assessed health status, lower BMI, and fewer chronic diseases) were positively associated with MHL. One possible reason for the more unfavorable health behavior of individuals with low MHL could be their reported difficulty in finding information on changing an unhealthy or maintaining a healthy lifestyle (Q17, Q20, and Q32). Without or with less knowledge on health behavior and healthy lifestyle, it may be difficult and hardly possible to change one's behavior or maintain a healthy lifestyle. Nevertheless, knowledge on health behavior or on changing lifestyle does not automatically lead to healthier behavior. In this context, there are certainly other factors, for example motivational or situational factors that may influence MHL and health behavior. Moreover, less healthy lifestyle of participants with low MHL was also associated with poor health outcome, and worse health status as well as higher occurrence of chronic diseases were associated with lower MHL.

\section{Implications}

Considering the present findings and the current substantial and increasing mental health burden, a measurement tool to assess and monitor MHL in a comprehensive approach is needed. The self-constructed MHL-Index of this study-based on the comprehensive model of HL-seems to be a promising first attempt. Additionally, although a correlation with general $\mathrm{HL}$ could be found, the present measure only included the domains finding and understanding of information on mental health issues. However, finding and understanding of such information without the ability of judging and applying it, is not sufficient to take responsibility for one's own mental and general health. In agreement with Mansfield et al. (10), it is therefore recommended that future MHL measures assess the ability to find, understand, appraise and to apply information on mental health to being able to take care of one's own mental health. Finally, the definition and assessment of MHL should comprise all relevant dimensions, including management of mental disorders, prevention thereof and also promotion of mental health. Thus, it could be recommended to extend the current HLS-questionnaire with an optional module including items capturing MHL across all these outlined domains and dimensions. The approach of incorporating current MHL constructs and definitions into a more holistic model may pave the way for a more unified research direction of MHL and HL in the future.

In addition, and in consideration of the limited MHL model used in this study, some first implications for public health in Zurich and Switzerland may be formulated as well. It could be revealed that almost half of the respondents showed low MHL levels. These levels might even be lower considering the missing assessment of the two domains appraising and applying of information on mental health. Moreover, the need to strengthen MHL in the general population might even be more important in respect to the increasing numbers of people with mental health problems, poor knowledge on management of mental health problems and difficulties in coping with this lack of knowledge. MHL could be strengthened by facilitating the access to information on mental health and to information which especially address topics like coping strategies for mental health problems and mental health promotion. In this context, destigmatization campaigns might play an important role in strengthening MHL as well. Anti-stigma interventions at the workplace for example have shown to be a promising approach by improving employees' knowledge and supportive behavior toward people with mental-health problems (54). Another promising option could be the initiation of tailormade interventions like mental health promotion campaigns for specific population groups. It seems to be crucial to increase their MHL and HL in order to strengthen their ability to care for their own mental and general health at the same time. In this context for example, Health Promotion Switzerland (55) has highlighted the importance of tailored community health education events on mental health and offers easy comprehensible health information for different target groups. Further initiations are however needed.

\section{Limitations}

There are certain methodological weaknesses that need to be considered when interpreting the present findings. First, due to the limited number of MHL-associated items, the present MHL-Index cannot be considered as a valid measure of a comprehensive MHL concept. Second, all data were self-reported and thereby carry the risk of reporting bias and social desirability. Additionally, quota and inclusion criteria may have only partially allowed for an unbiased selection of participants, as interviewers were free to choose the location of recruitment. Third, the use of German language only might have excluded people less competent in this language and might have led to a selection 
bias. Regarding data analysis, the transformation of ordinal and continuous into categorial data could have led to an exclusion of important information, despite the advantages of the multiple logistic regression as an adjusted analysis method. In addition, listwise exclusion led to reduced group sizes and might have affected the informative value, e.g., in the groups rural residence, poor self-rated health status or low education. Therefore, spearmen rank's correlation has been valued higher, as results were independent of categorizations and based on more individuals. Moreover, more information could be considered by including ordinal and continuous data. For future approaches and when statistical assumptions can be fulfilled, it might be recommended to rather make use of a linear regression method, or to make sure to include enough respondents for each category. Also, Cronbach's alpha of the MHL-associated items was quite low. This had to be expected because the items were not selfgenerated or composed to measure a predefined construct, nor did the index contain a great number of items. However, the single MHL-associated items were created in a logical, systematic and structured development process (31) and gave important insights into MHL of the population of the canton of Zurich. Finally, regarding the aspect of mental health, the present survey assessed rather unspecific information on health behavior and health outcome. Therefore, future MHL surveys should capture such variables more specifically, for example by asking for specific mental diseases, drug consumption, and addictions.

\section{CONCLUSIONS}

The present study gives first insights into several aspects of MHL among residents of the canton of Zurich using an adapted version of the commonly used health literacy survey HLS-EUQ47. A substantial number of individuals reported difficulties in handling information on mental health, which in turn was associated with lower HL, less favorable health behavior and poorer health outcome. Therefore, especially in times of a pandemic and increasing mental health burden, it seems important to identify residents' MHL deficits. Based on these findings, they should be supported in their access, understanding, assessing and applying of information on mental health as well as their resilience to stress and other mental health issues and the promotion of their mental well-being. To capture MHL in a more comprehensive manner, the HLS-EU-Q47 could be extended by considering recent MHL constructs and definitions, and including all domains (finding, understanding, appraising, and applying information on mental health) and dimensions

\section{REFERENCES}

1. World Health Organization. The WHO Special Initiative for Mental Health (2019-2023): Universal Health Coverage for Mental Health. (2019). Available online at: https://apps.who.int/iris/handle/10665/310981 (accessed May 15, 2021).

2. World Health Organization. Investing in Mental Health. Geneva: World Health Organization (2003). p. 1-13. (management of mental disorders, prevention thereof, and promotion of mental health).

\section{DATA AVAILABILITY STATEMENT}

The datasets for this study are the property of the Careum Foundation and the Department of Health of the Canton of Zurich and can be received on reasoned request.

\section{ETHICS STATEMENT}

Ethical review and approval was not required for the study on human participants in accordance with the local legislation and institutional requirements. Written informed consent for participation was not required for this study in accordance with the national legislation and the institutional requirements.

\section{AUTHOR CONTRIBUTIONS}

MS, SD, and DN-F conceptualized this study, developed the research question, and were responsible for project administration. MS performed the data analysis and literature research. MS, SD, and RJ prepared the original draft of the article. DN provided critical review of the concept and the manuscript. All authors revised it critically, approved the final manuscript and agreed to be personally accountable for their own contribution to the article and to ensure the accuracy or integrity of any part of the work.

\section{FUNDING}

Data was collected in the course of the Health Literacy Survey Zurich 2018 which was realized by the Careum Foundation and the Department of Health of the Canton of Zurich.

\section{ACKNOWLEDGMENTS}

This project was part of a master thesis (not published so far). In this context, we would like to acknowledge gfs.bern AG and especially Jonas Kocher for providing detailed information on the data collection process and their statistical advice. Moreover, we are grateful for the statistical advice provided by the statistical consulting service of ETH Zurich. We would also like to thank Prof. Eling D. de Bruin and the interview partners for their cooperation.
3. Schuler D, Tuch A, Peter C. Psychische Gesundheit in der Schweiz Monitoring 2020. Neuchâtel: Schweizerisches Gesundheitsobservatorium (2020) p. 4-6.

4. de Quervain D, Aerni A, Amini E, Bentz D, Coynel D, Freytag V, et al. The Swiss Corona Stress Study: Second Pandemic Wave, November 2020. (2020). Available online at: https://osf.io/6cseh/ (accessed May 14, 2021).

5. Kelly CM, Jorm AF, Wright A. Improving mental health literacy as a strategy to facilitate early intervention for mental disorders. Med J Aust. (2007) 187:26-30. doi: 10.5694/j.1326-5377.2007.tb01332.x 
6. Bjørnsen, HN. Promoting Adolescent Mental Health - Positive Mental Health Literacy as a Concept and Working Strategy for School Health Services (dissertation). Trondheim: Norwegian University of Science and Technology (2019).

7. Sørensen K, Van Den Broucke S, Fullam J, Doyle G, Pelikan J, Slonska Z, et al. Health literacy and public health: a systematic review and integration of definitions and models. BMC Public Health. (2012) 12:80. doi: 10.1186/1471-2458-12-80

8. Malloy-Weir LJ, Charles C, Gafni A, Entwistle V. A review of health literacy: definitions, interpretations, and implications for policy initiatives. J Public Health Policy. (2016) 37:334-352. doi: 10.1057/jphp.2016.18

9. Spiker DA, Hammer JH. Mental health literacy as theory: current challenges and future directions. J Ment Health. (2019) 28:238-42. doi: 10.1080/09638237.2018.1437613

10. Mansfield R, Patalay P, Humphrey N. A systematic literature review of existing conceptualisation and measurement of mental health literacy in adolescent research: current challenges and inconsistencies. BMC Public Health. (2020) 20:607. doi: 10.1186/s12889-020-08734-1

11. Jorm AF, Korten AE, Jacomb PA, Christensen H, Rodgers B, Pollitt P. "Mental health literacy": a survey of the public's ability to recognise mental disorders and their beliefs about the effectiveness of treatment. Med J Aust. (1997) 166:182-6. doi: 10.5694/j.1326-5377.1997.tb140071.x

12. Bjørnsen HN, Eilertsen MB, Ringdal R, Espnes GA, Moksnes UK. Positive mental health literacy: development and validation of a measure among Norwegian adolescents. BMC Public Health. (2017) 17:717. doi: 10.1186/s12889-017-4733-6

13. Kutcher S, Wei Y, Coniglio C. Mental health literacy: past, present, and future. Can J Psychiatry. (2016) 61:154-8. doi: 10.1177/0706743715616609

14. Kusan S. Dialectics of mind, body, and place: groundwork for a theory of mental health literacy. SAGE Open. (2013) 3:1-16. doi: 10.1177/2158244013512131

15. World Health Organization. Preamble to the Constitution of the World Health Organization as Adopted by the International Health Conference, New York, NY, USA, 19-22 June 1946 Signed on 22 July 1946 by the Representatives of 61 States (Official Records of the World Health Organization, no. 2, p.100) and Entered into Force on 7 April 1948. (1948). Available online at: http://www. who.int/governance/eb/who_constitution_en.pdf (accessed May 14, 2021).

16. HLS-EU Consortium. Comparative Report of Health Literacy in Eight EU Member States. The European Health Literacy Survey (Second Revised and Extended Version) (2014). Available online at: https://cdn1.sph.harvard.edu/ wp-content/uploads/sites/135/2015/09/neu_rev_hls-eu_report_2015_05_ 13_lit.pdf (accessed May 14, 2021).

17. Bieri U, Kocher JP, Gauch C, Tschöpe S, Venetz A, Hagemann M et al. Bevölkerungsbefragung "Erhebung Gesundheitskompetenz 2015" Schlussbericht. (2016). Available online at: https://www.bag.admin. $\mathrm{ch} / \mathrm{bag} / \mathrm{de} / \mathrm{home} /$ strategie-und-politik/nationale-gesundheitspolitik/ gesundheitskompetenz.html (accessed May 14, 2021).

18. Fleary SA, Joseph P, Pappagianopoulos JE. Adolescent health literacy and health behaviors: a systematic review. J Adolesc. (2018) 62:11627. doi: 10.1016/j.adolescence.2017.11.010

19. Buja A, Rabensteiner A, Sperotto M, Grotto G, Bertoncello C, Cocchio S, et al. Health literacy and physical activity: a systematic review. J Phys Act Health. (2020) 17:1259-74. doi: 10.1123/jpah.2020-0161

20. Berkman ND, Sheridan SL, Donahue KE, Halpern DJ, Crotty K. Low health literacy and health outcomes: an updated systematic review. Ann Intern Med. (2011) 155:97-107. doi: 10.7326/0003-4819-155-2-201107190-00005

21. Reavley NJ, Morgan AJ, Jorm AF. Development of scales to assess mental health literacy relating to recognition of and interventions for depression, anxiety disorders and schizophrenia/psychosis. Aust N Z J Psychiatry. (2014) 48:61-9. doi: 10.1177/0004867413491157

22. Lee HY, Hwang J, Ball JG, Lee J, Albright DL. Is health literacy associated with mental health literacy? Findings from Mental Health Literacy Scale. Perspect Psychiatr Care. (2020) 56:393-400. doi: 10.1111/ppc.12447

23. Bjørnsen HN, Espnes GA, Eilertsen MB, Ringdal R, Moksnes UK. The relationship between positive mental health literacy and mental well-being among adolescents: implications for school health services. J Sch Nurs. (2019) 35:107-16. doi: 10.1177/1059840517732125
24. Schroeder S, Tan CM, Urlacher B, Heitkamp T. The role of rural and urban geography and gender in community stigma around mental illness. Health Educ Behav. (2021) 48:63-73. doi: 10.1177/1090198120974963

25. Hurley D, Swann C, Allen MS, Ferguson HL, Vella SA. A systematic review of parent and caregiver mental health literacy. Community Ment Health J. (2020) 56:2-21. doi: 10.1007/s10597-019-00454-0

26. Cheesmond NE, Davies K, Inder KJ. Exploring the role of rurality and rural identity in mental health help-seeking behavior: a systematic qualitative review. Rural Ment Health. (2019) 43:45-59. doi: 10.1037/rmh0000109

27. McCann TV, Mugavin J, Renzaho A, Lubman DI. Sub-Saharan African migrant youths' help-seeking barriers and facilitators for mental health and substance use problems: a qualitative study. BMC Psychiatry. (2016) 16:275. doi: 10.1186/s12888-016-0984-5

28. Reavley NJ, Cvetkovski S, Jorm AF, Lubman DI. Help-seeking for substance use, anxiety and affective disorders among young people: results from the 2007 Australian National Survey of Mental Health and Wellbeing. Aust N Z J Psychiatry. (2010) 44:729-35. doi: 10.3109/00048671003705458

29. Kim YS, Lee HY, Lee MH, Simms T, Park BH. Mental health literacy in korean older adults: a cross-sectional survey. J Psychiatr Ment Health Nurs. (2017) 24:523-33. doi: 10.1111/jpm.12395

30. Lam LT. Mental health literacy and mental health status in adolescents: a population-based survey. Child Adolesc Psychiatry Ment Health. (2014) 8:26. doi: 10.1186/1753-2000-8-26

31. Sørensen K, Van den Broucke S, Pelikan JM, Fullam J, Doyle G, Slonska $Z$, et al. Measuring health literacy in populations: illuminating the design and development process of HLS-EU-Q. BMC Public Health. (2013) 13:948. doi: 10.1186/1471-2458-13-948

32. Vogt D, Gehrig SM. Bedeutung und Stärkung von Gesundheitskompetenz/Health Literacy in der Prävention und Gesundheitsförderung. In: Tiemann M, Mohokum M, editors. Prävention und Gesundheitsförderung. Berlin; Heidelberg; Deutschland: Springer (2020). p. $1-11$.

33. Sørensen K, Pelikan JM, Röthlin F, Ganahl K, Slonska Z, Doyle G, et al. Health literacy in Europe: comparative results of the European health literacy survey (HLS-EU). Eur J Public Health. (2015) 25:1053-8. doi: 10.1093/eurpub/ckv043

34. UNESCO Institute for Statistics. International Standard Classification of Education: ISCED. Montreal: UNESCO Institute for Statistics (1997). p. $14-39$.

35. Bundesamt für Statistik. Ständige und Nichtständige Wohnbevölkerung nach Institutionellen Gliederungen, Staatsangehörigkeit (Kategorie), Geschlecht und Alter. (2020). Available online at: http://www.pxweb.bfs.admin.ch/pxweb/de/ px-x-0102010000_101/-/px-X-0102010000_101.px/(accessed May 7, 2021).

36. Bundesamt für Statistik. Ständige Wohnbevölkerung ab 15 Jahren Nach Höchster Abgeschlossener Ausbildung und Kanton. (2018). Available online at: https://www.bfs.admin.ch/bfs/de/home/statistiken/kataloge-datenbanken/ tabellen.assetdetail.4242914.html (accessed May 9, 2021).

37. Schnyder N, Panczak R, Groth N, Schultze-Lutter F. Association between mental health-related stigma and active help-seeking: systematic review and meta-analysis. BJPsych. (2017) 210:261-8. doi: 10.1192/bjp.bp.116.189464

38. Gesundheitsförderung Schweiz. Weiss Die Schweiz, was sie für ihre psychische Gesundheit tun kann? Ergebnisse einer Repräsentativen Befragung zur Psychischen Gesundheitskompetenz in der Schweiz. (2021). Available online at: https://gesundheitsfoerderung.ch/assets/public/documents/ de/5-grundlagen/publikationen/psychische-gesundheit/faktenblaetter/ Faktenblatt_060_GFCH_2021-06_-_Psychische_Gesundheitskompetenz_ in_der_Schweiz.pdf (accessed July 19, 2021).

39. Dey M, Marti L, Venzin V. Schweizer Befragung von Jugendlichen/jungen Erwachsenen zur psychischen Gesundheitskompetenz und Stigma. (2018). doi: $10.5167 /$ uzh-153031

40. Geboers B, Uiters E, Reijneveld SA, Jansen CJM, Almansa J, Nooyens ACJ, et al. Health literacy among older adults is associated with their 10-years' cognitive functioning and decline - the Doetinchem Cohort Study. BMC Geriatr. (2018) 18:77. doi: 10.1186/s12877-018-0766-7

41. Turner AM, Osterhage KP, Taylor JO, Hartzler AL, Demiris G. A closer look at health information seeking by older adults and involved family and friends: design considerations for health information technologies. AMIA Annu Symp Proc. (2018) 2018:1036-45. 
42. Graham N, Lindesay J, Katona C, Bertolote JM, Camus V, Copeland JRM, et al. Reducing stigma and discrimination against older people with mental disorders: a technical consensus statement. Int J Geriat Psychiatry. (2003) 18:670-8. doi: 10.1002/gps.876

43. Martino H, Rabenschlag F, Koch U, Attinger-Andreoli Y, Michel K, Gassmann J, et al. Arbeitspapier Entstigmatisierung. (2012). Available online at: https://www.google.com/url?sa= $\mathrm{t} \& \mathrm{rct}=\mathrm{j} \& \mathrm{q}=\& \mathrm{esrc}=\mathrm{s} \&$ source $=$ web $\& \mathrm{~cd}=\& \mathrm{cad}=\mathrm{rja} \& u a c t=8 \& \mathrm{ved}=$ 2ahUKEwjM5sTa9I3xAhVCmqQKHe5JA5wQFjAAegQIBBAD\& url=https $\% 3 \mathrm{~A} \% 2 \mathrm{~F} \% 2$ Fpublic-health.ch\%2Fdocuments $\% 2 \mathrm{~F} 232$ \%2FEntstigmatisierung_Arbeitspapier_DEF.pdf\&usg=AOvVaw1tpdWXeQ9hP8hpwvBFOE2 (accessed April 14, 2021).

44. Schuler D, Tuch A, Buscher N, Camenzind P. Psychische Gesundheit in der Schweiz Monitoring 2016. Neuchâtel: Schweizerisches Gesundheitsobservatorium (2016). p. 61-4.

45. Baer N, Schuler D, Füglister-Dousse S, Moreau-Gruet F. Depressionen in der Schweizer Bevölkerung. Daten zur Epidemiologie, Behandlung und sozial-beruflichen Integration. Neuchâtel: Schweizerisches Gesundheitsobservatorium (2013). p. 27-44.

46. Bundesamt für Statistik. Mittlere oder hohe psychische Belastung, 2017. (2018). Available online at: https://www.bfs.admin.ch/bfs/de/home/ statistiken/gesundheit/gesundheitszustand/psychische.assetdetail.6466168. html (accessed May 4, 2021).

47. Corrigan PW, Watson AC. The stigma of psychiatric disorders and the gender, ethnicity, and education of the perceiver. Community Ment Health J. (2007) 43:439-58. doi: 10.1007/s10597-007-9084-9

48. Yin H, Wardenaar KJ, Xu G, Tian H, Schoevers RA. Mental health stigma and mental health knowledge in Chinese population: a cross-sectional study. BMC Psychiatry. (2020) 20:323. doi: 10.1186/s12888-020-02705-x

49. Coles ME, Schubert JR, Heimberg RG, Weiss BD. Disseminating treatment for anxiety disorders: Step 1: recognizing the problem as a precursor to seeking help. J Anxiety Disord. (2014) 28:737-40. doi: 10.1016/j.janxdis.2014. 07.011

50. Coles ME, Heimberg RG, Weiss BD. The Public's knowledge and beliefs about obsessive compulsive disorder. Depress Anxiety. (2013) 30:77885. doi: $10.1002 /$ da. 22080
51. Wang J, He Y, Jiang Q, Cai J, Wang W, Zeng Q, et al. Mental health literacy among residents in Shanghai. Shanghai Arch Psychiatry. (2013) 25:22435. doi: 10.3969/j.issn.1002-0829.2013.04.004

52. Bundesamt für Statistik. Soziale Situation. (2019). Available online at: https:// www.bfs.admin.ch/bfs/de/home/statistiken/gesundheit/determinanten/ soziale-situation.html (accessed May 4, 2021).

53. Lund C, Breen A, Flisher AJ, Kakuma R, Corrigall J, Joska JA, et al. Poverty and common mental disorders in low and middle income countries: A systematic review. Soc Sci Med. (2010) 71:517-28. doi: 10.1016/j.socscimed.2010.04.027

54. Hanisch SE, Twomey CD, Szeto ACH, Birner UW, Nowak D, Sabariego C. The effectiveness of interventions targeting the stigma of mental illness at the workplace: a systematic review. BMC Psychiatry. (2016) 16:1. doi: 10.1186/s12888-015-0706-4

55. Jacobshagen N. Lebenskompetenzen und psychische Gesundheit im Alter. (2020). Available online at: https://www.npg-rsp.ch/fileadmin/npg-rsp/ Themen/Fachthemen/GFCH_2020_Lebenskompetenzen_und_psychische_ Gesundheit_im_Alter.pdf (accessed July 19, 2021).

Conflict of Interest: The authors declare that the research was conducted in the absence of any commercial or financial relationships that could be construed as a potential conflict of interest.

Publisher's Note: All claims expressed in this article are solely those of the authors and do not necessarily represent those of their affiliated organizations, or those of the publisher, the editors and the reviewers. Any product that may be evaluated in this article, or claim that may be made by its manufacturer, is not guaranteed or endorsed by the publisher.

Copyright (C) 2021 Schneider, Jaks, Nowak-Flück, Nicca and De Gani. This is an open-access article distributed under the terms of the Creative Commons Attribution License (CC BY). The use, distribution or reproduction in other forums is permitted, provided the original author(s) and the copyright owner(s) are credited and that the original publication in this journal is cited, in accordance with accepted academic practice. No use, distribution or reproduction is permitted which does not comply with these terms. 